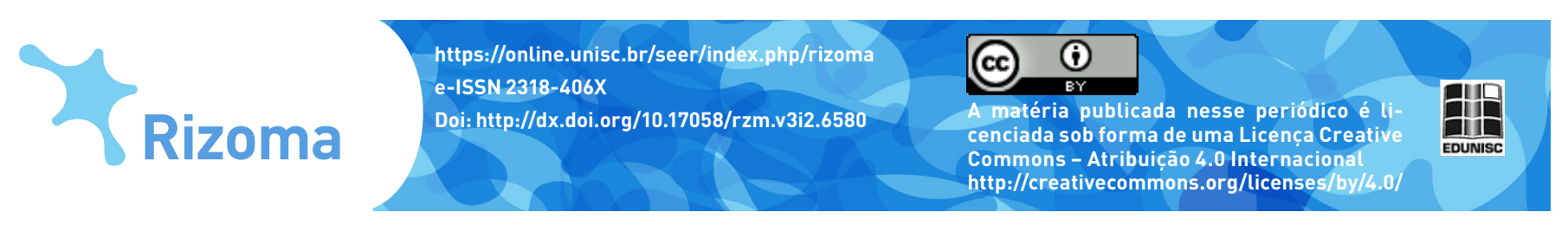

\title{
Ingeniería en comunicación social y opinión pública. Apunte a partir de la propuesta de Eulalio Ferrer
}

\author{
Jesús Galindo Cáceres ${ }^{1}$
}

\section{Resumen}

El texto se compone de tres partes. En la primera se presenta un apunte sintético sobre la propuesta general de la Comunicología y la Ingeniería en Comunicación Social, poniendo énfasis en las figuras conceptuales de los sistemas de información y los sistemas de comunicación. En la segunda parte se presenta una síntesis del libro "Comunicación y Opinión Pública", de Eulalio Ferrer, en diez puntos, poniendo énfasis en la configuración constructiva del concepto de Opinión Pública, considerada como matriz socio cultural de la vida colectiva. En la tercera parte se explora la construcción de una posible Ingeniería en Comunicación Social de la Opinión Pública, relacionando la primera parte con la segunda. Partiendo de los conceptos de sistema de información y sistema de comunicación, y de las figuras constructivas de difusión e interacción, se explora modelo de comunicación social de la opinión pública propuesta por Eulalio Ferrer que tiene a la democracia en el centro, y que puede ser leída por la Comunicología como un sistema de comunicación social interacción colaboración ciudadana.

Palabras claves: Opinión Pública; Comunicología; Ingeniería en Comunicación Social; Sistemas de Información; Sistemas de Comunicación; Difusión; Interacción.

\section{Resumo}

O texto é composto por três partes. Na primeira nota, a síntese sobre a proposta geral "Comunicologia e Engenharia de Comunicação Social" é apresentada, enfatizando as figuras conceituais de sistemas de informação e sistemas de comunicação. Na segunda parte, há um resumo do livro "Comunicação e Opinião Pública», de Eulalio Ferrer, em dez pontos, enfatizando o projeto estrutural do conceito de opinião pública, matriz sociocultural considerada de vida coletiva. Na parte três, uma possível construção de Engenharia de Comunicação Social de Opinião Pública é explorada, relacionando a primeira parte com a segunda. Com base nos conceitos de sistema de informação e sistema de comunicação e construção de figuras de difusão e interação, modelo de comunicação social proposta por Eulalio Ferrer tendo a democracia

\footnotetext{
${ }^{1}$ Mexicano. Doctor en Ciencias Sociales, Doctor en Comunicación. Autor de 35 libros y más de trescientos cincuenta artículos académicos publicados en catorce países de América y Europa. Promotor cultural en diversos proyectos desde 1972. Profesor en Argentina, Brasil, Colombia, Perú, Ecuador, España y México desde 1975. Miembro del Programa de Estudios sobre las Culturas Contemporáneas desde 1985. Miembro del Sistema Nacional de Investigadores SNI-CONACYT desde 1987. Promotor del Grupo de Acción en Cultura de Investigación (GACI) desde 1994. Promotor de la Red de estudios en teoría de la comunicación (REDECOM) y del Grupo hacia una Comunicología posible (GUCOM) desde el 2003.

Miembro de la Red Deporte, Cultura Física, Ocio y Recreación desde 2006. Promotor del Grupo Ingeniería en Comunicación Social (GICOM) desde el 2009. Miembro de la Red Iberoamericana de Gestores Culturales desde el 2009. Miembro del Instituto en Altos Estudios sobre Deporte, Cultura y Sociedad (INDECUS) desde el 2010. Trabaja en el Centro de Estudios en Comunicación Política, del Instituto de Ciencias del Gobierno y Desarrollo Estratégico, Benemérita Universidad Autónoma
} de Puebla. 

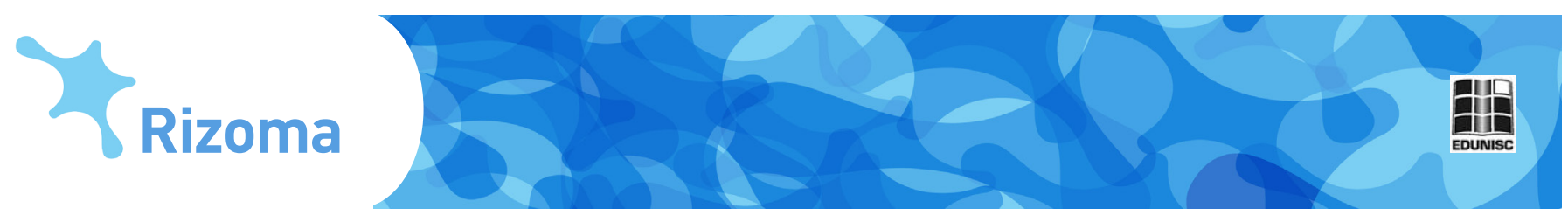

no centro, explora a opinião pública. E pode ser Comunicologia lida por um sistema de comunicação social, cidadão, colaborativo e interativo.

Palavras-chave: Opinião Oública; Comunicologia; Engenharia de Comunicação Social; Sistemas de Informação; Sistemas de Comunicação; Difusão; Interação.

\begin{abstract}
The text consists of three parts. In the first synthetic note on the general proposal Communicology and Social Communication Engineering is presented, emphasizing the conceptual figures of information systems and communication systems. In the second part a summary of the book "Communication and Public Opinion," Eulalio Ferrer, in ten points, emphasizing the structural design of the concept of public opinion, considered sociocultural matrix of collective life occurs. In part three of a possible construction Engineering in Social Communication of Public Opinion is explored by relating the first part with the second. Based on the concepts of information system and communication system, and building figures diffusion and interaction, social communication model proposed by Eulalio Ferrer having to democracy at the center explores public opinion, and may be Communicology read by a system of social communication interaction citizen collaboration.
\end{abstract}

Keywords: Public Opinion; Communicology; Social Communication Engineering; Information Systems; Communication Systems; Diffusion; Interaction.

\title{
1. Comunicología e Ingeniería en Comunicación Social
}

Una ciencia de la comunicación es posible, su nombre Comunicología. Esta perspectiva constructiva de una visión del mundo y la vida social se funda en un concepto general, y a partir de ahí configura una matriz de conceptos que en forma inicial forman la guía de una ciencia que se desarrolla y complejiza.

El concepto general de comunicación parte de su definición elemental etimológica, comunicación es la acción de poner en común. Es la acción, esta pauta define la naturaleza del concepto y de su utilidad, no se trata de un estado, de sólo una situación, se trata del movimiento que construye a un estado y detona el contenido y proceso de una situación. Puesta en común, ese es el horizonte mismo del sentido de este concepto, la formación de comunidad, la articulación de los separados y los diversos, de los distintos 
y los aislados, de los opuestos y los alternos. La acción de configurar una comunidad en el universo de lo posible.

Desde el proyecto de trabajo del GUCOM, Grupo hacia una Comunicología Posible, a lo largo de la primera década del siglo veinte, se estabilizó una matriz que contenía siete fuentes básicas del pensamiento científico en comunicación, fuentes de origen sociológico, psicológico, lingüístico, semiótico, económico, político, cibernético. En un ensayo epistemológico constructivo las dos que quedaron al centro por su nivel de configuración lógico-formal fueron la Semiótica y la Cibernética. Y por otra parte, en la dimensión científica más apegada a la tierra y lo particular, se ubicó a la Sociología Fenomenológica y a la Psicología Social. De esta multi articulación nace una ciencia general de la comunicación posible, con el pensamiento cibernético y semiótico como guía, con el compromiso metodológico de conjuntar sus abstracciones en el piso cotidiano de la comunicación del día a día y de la acción convencional.

De todo ello nacieron tres puntos de configuración general de la comunicación social.-

1) Primero. Los conceptos centrales son la información y la comunicación, y con ellos se construye una doble apreciación del mundo social como sistemas de información y sistemas de comunicación. Los primeros representando a todo aquello que nos prescribe desde el pasado hacia el presente, producto de acciones que han estabilizado formas de comunión código, que indican lo que puede y lo que no puede suceder y el sentido que eso tiene, los segundos representando lo que posibilita la creación social del presente hacia el futuro, a partir de las acciones e interacciones que entrelazan, combinan, enactivan, reproducen y modifican los sistemas de información. Nuestro comportamiento presente y su sentido deviene de los sistema de información que los prescriben. La vida de esos sistemas de información en el presente, afectándose unos a otros o determinándose unos a otros, es lo que configura los sistemas de comunicación, que pueden reproducir el pasado, o ser el punto de quiebre, modificación, alteración hacia el futuro. La energía del presente en la forma del pasado hacia el futuro, o en la nueva forma del futuro desde el presente.

2) Segundo. Los sistemas de información tienen dos tipos de asociación básica en sistemas de comunicación, la difusión y la interacción. La Difusión muestra cuando un sistema de información se mueve para poner en su forma a otro sistema de información. La Interacción muestra cuando dos o más sistemas de información se ponen en contacto para mutuamente modificarse o enriquecerse, con la cualidad que todos los sistemas de información se alteran por la acción de los otros, modificando su forma por la presencia e intervención de los demás, la intención no es que uno solo modifique a 

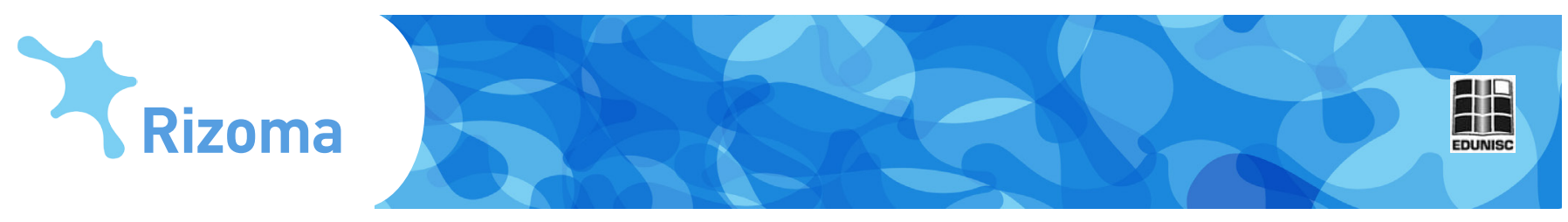

todos los otros.

3) Tercero. Cuando los sistemas de información entran en contacto mediante la difusión, lo que tiende a acontecer es la configuración de un sistema de comunicación dominación. Cuando los sistemas de información entran en contacto mediante la interacción lo que tiende a acontecer es la configuración de un sistema de comunicación colaboración.

Con estos tres puntos de configuración general la Comunicología realiza, junto con otros elementos de percepción comunicológica, una lectura de la vida social, construyendo en forma metodológico-conceptual las figuras de los sistemas de información que están operando para constituir el presente, $\mathrm{y}$ en forma complementaria identificando de igual forma las figuras de los sistemas de comunicación que posibilitan el futuro.

La Ingeniería en Comunicación Social lo que hace con esta lectura comunicológica es cumplir con su primer momento metodológico general, el diagnóstico de una situación social desde la perspectiva de la comunicación. Y con ello se abre el segundo momento metodológico general, la síntesis de operaciones de intervención o acompañamiento en esa situación social así leída en un presente posible, a partir de un pasado probable, y un futuro probable y posible.

La Ingeniería en Comunicación Social opera en el horizonte de tres escenarios posibles. Empoderar el movimiento constructivo del pasado hacia el futuro a partir del cierto presente, debilitar ese movimiento constructivo, o apoyar su sustentabilidad. La vida social de esta manera es leída en su configuración dinámica como un haz de trayectorias, movimiento del pasado hacia el presente, y como un haz de tendencias, movimiento del presente hacia el futuro. Todo ello en la articulación de sistemas de información, movimiento pasado hacia el presente, en la configuración de sistemas de comunicación, movimiento del presente hacia el futuro.

En este sentido es posible diagnosticar las trayectorias que han llevado a la situación presente de cierto tipo de configuración de la opinión pública, al tiempo que se trazan las tendencias que mueven el presente hacia cierto tipo de opinión pública en el futuro. Las operaciones de Ingeniería Social pueden dar sustentabilidad a cierto movimiento social del pasado hacia el futuro, así como modificar esa sustentabilidad reforzando o debilitando ciertas operaciones que articulan de cierta forma a los sistemas de información y a los sistemas de comunicación.

\section{Opinión Pública en Eulalio Ferrer}

Para proponer un apunte sobre Ingeniería en Comunicación Social de la Opinión Pública el libro sobre el tema de Eulalio Ferrer será el referente, “Comunicación y Opinión Pública. La comunicación cambia o modifica el 
semblante del mundo". El texto arma su argumentación desde un concepto de comunicación generoso y optimista, pasando por una revisión de temas y asuntos asociados a la Psicología Social y a la Sociología de las colectividades sociales, enfatizando el papel que los medios de difusión tienen en toda la configuración.

El libro de Eulalio Ferrer se compone de seis capítulos, un prólogo y un epílogo. El primer capítulo presenta un concepto general de comunicación. El segundo, una primera definición general de Opinión Pública. El tercero, la caracteriza. El cuarto presenta a los medios masivos como un dispositivo clave en la configuración de la opinión pública en la vida contemporánea de la comunicación social. El quinto hace una referencia general a los conceptos de información y comunicación para mejor entender a la comunicación social y la opinión pública. Y el sexto concluye con la propuesta central del libro sobre la articulación de la opinión pública y la comunicación social. A continuación un esquema sintético del libro, y un primer comentario sobre su composición y organización de argumentos.

Esquema sintético del libro de Comunicación y Opinión Pública de Eulalio Ferrer, en un apunte en diez puntos.-

1. Estamos ante una nueva era en la historia humana, la formación de la comunidad global. Comunicación es formación de comunidad.

2. La opinión pública es la expresión de un consenso mayoritario. Hay que distinguir entre opinión pública y opinión del público, la primera es cultura, es estable, matricial, a largo plazo, la segunda es coyuntural, la expresión de la mayoría, o de una parte del colectivo social, en un momento dado, bajo ciertas circunstancias y condiciones.

3. La opinión pública se forma en principio por diversos agentes y componentes de su difusión y confirmación, el más importante es el de la propaganda, que se ejerce en principio y en forma mayoritaria por parte del gobierno constituido en forma democrática.

4. Los medios de difusión colectiva tienen un papel central en la formación de la opinión pública, son instrumentos básicos de influencia y equilibrio social. Los medios sobre todo refuerzan a la opinión pública, su efecto en principio no es de cambio radical sino de mantenimiento.

5. Los medios de difusión colectiva representan un enorme poder, vivimos en este momento el tiempo social de su ejercicio y presencia. Su poder deriva sobre todo de su articulación social con la vida cotidiana en extenso y con cierta intensidad.

6. La institucionalización de la opinión pública es lo que permite concebirla en plenitud, es el efecto de una suma de presencia cotidiana de los medios y su propaganda y de la mayor estabilidad 


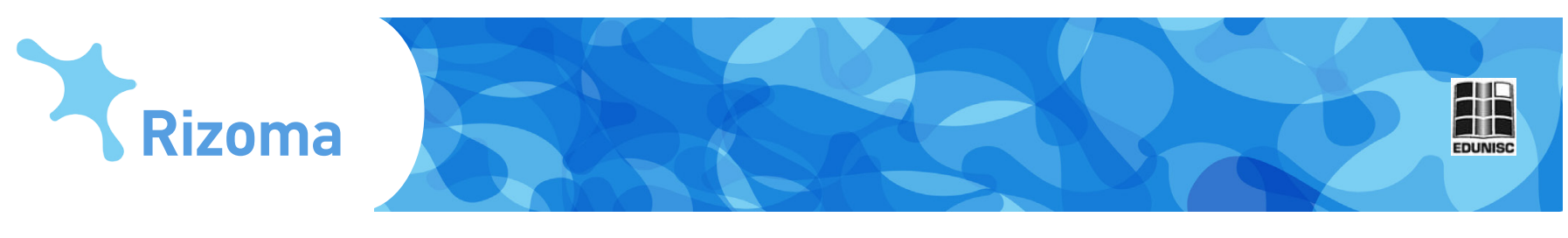

social. La opinión pública se conforma en una fórmula que combina medios y estabilidad.

7. En principio los medios de difusión refuerzan la opinión pública mayor, general, y por efecto modifican la pequeña, la menos profunda, la más superficial, la menos arraigada. En este sentido hay una opinión pública más dependiente y otra más independiente del efecto de los medios, la más importante y mayoritaria es más independiente, la asociada a la cultura, a la matriz social básica, y la más dependiente es la asociada a lo coyuntural, a la agenda del día a día, a la moda pasajera, a la noticia circunstancial.

8. El tema de fondo en el marco del punto anterior es la relación entre el cambio y la tradición. La relación de la información con las matrices de fondo de la cultura y el sentido general de la vida social, y la relación de la información con los datos, las peculiaridades que pueden percibirse sin la necesidad a priori de la articulación de fondo. La opinión pública es articulación de fondo, la opinión del público es articulación de superficie. La relación entre ambas es un asunto central en el estudio y la conformación del fenómeno.

9. El tema más agudo en los asuntos de la formación y desarrollo de la opinión pública es el de la libertad de expresión frente a la indiferencia y la apatía. La vida ciudadana se configura en una combinación de opiniones particulares asociadas a discursos generales, la conversación es clave. El gran poder de la opinión pública es la conversación pública de agendas permanentes y coyunturales. El ideal es la constante y rica conversación libre, ilustrada, comprometida y responsable. Pero esta condición puede ser escasa, entonces el asunto de la opinión pública tiene los matices propios de la necesidad de su formación, la pregunta entonces es qué hacer para educar y promover en esa cultura de conversación democrática.

10. La opinión pública necesita de la opinión publicada, es decir de la expresión y el debate ciudadano, sin esta configuración sus condiciones de desarrollo son improbables. La comunicación, formación de comunidad, parte de la condición elemental de la conversación democrática. La conversación es el componente básico para el desarrollo y evolución de la opinión pública.

La propuesta de Eulalio Ferrer sobre la opinión pública parte del concepto de comunicación como formación de comunidad, se articula en una figura que asimila a la opinión pública con la matriz socio cultural general de la colectividad, se hace operativa en la oposición complementaria entre lo coyuntural y lo más estable, entre lo general y lo particular, entre la tradición y el cambio, y adquiere una pertinencia constructiva con la operación de los medios de difusión colectiva como los reforzadores de lo 
matricial, y los difusores de lo coyuntural, ante el tejido conversacional de la vida ciudadana. La opinión pública entonces se configura en el orden y organización de la cultura y se desarrolla en la dinámica conversacional de las agendas ciudadanas y de gobierno, mediada por la operación de los medios de difusión colectiva.

\section{Ingeniería en Comunicación Social y Opinión Pública}

Ahora una nota sobre la lectura de Eulalio Ferrer sobre la opinión pública siguiendo el pequeño apunte de Ingeniería en Comunicación Social presentado en la primera parte. El apunte se centraba en tres puntos, las figuras conceptuales generales de la información y la comunicación, dentro del espacio comunicológico elemental de los conceptos de sistema de información y sistema de comunicación, la propuesta analítica primaria de las dimensiones de difusión e interacción y la dicotomía constructiva comunicológica básica de los sistemas de comunicación dominación y los sistemas de comunicación colaboración. Con este marco será revisada la propuesta de Eulalio Ferrer en los próximos párrafos.

1. El concepto de comunicación. La figura de comunidad está asociada dentro de la comunicología social básica con las figuras del sistema de comunicación interacción colaboración. No sólo sucede así, pero la connotación central de comunidad coincide con esta configuración de sistema de comunicación. La comunidad es un espacio en cual los diversos sistemas de información se ponen en contacto, interactúan y se enriquecen mutuamente para formar un todo más complejo, que a su vez supone la búsqueda de nuevos sistemas de información y comunicación con los cuales interactuar y colaborar.

2. La opinión pública es la expresión de un consenso mayoritario. Hay que distinguir entre opinión pública y opinión del público. La opinión pública corresponde con el sistema de comunicación social general de una comunidad-asociación determinada. El punto clave es que esa forma social particular percibida comunicológicamente como sistema de comunicación, tiene en su seno diversos sistemas de información en contacto e interacción, de diversa magnitud y complejidad, en distintos niveles de composición de lo social. De todos ellos hay uno que gobierna el sentido común, ese sistema de información general articulado y articulando a la diversidad es la opinión pública. El sistema de comunicación social total gobernado en lo general por ese sistema de información articulador del consenso está cerca de la noción de opinión pública de Eulalio Ferrer. La opinión del público es un tipo de manifestación de ese sistema de información general dentro del 


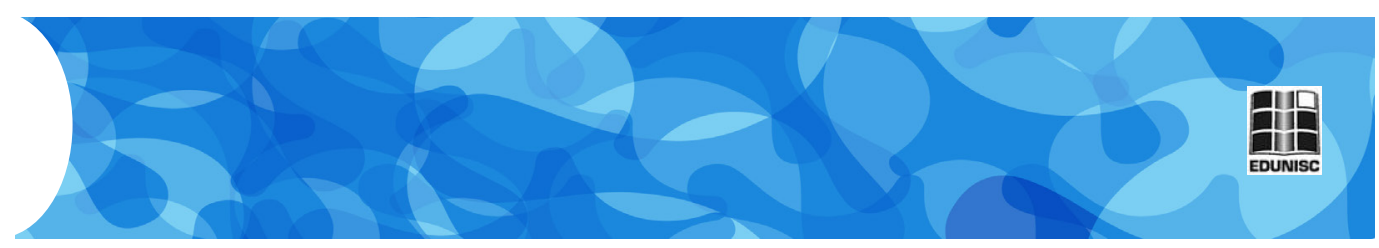

sistema de comunicación general, que en una situación presente puede tener ciertas motivaciones y resultar del efecto de la acción de otros sistemas de información menores o coyunturales.

3. Opinión Pública, Propaganda y Democracia. Este es un punto que hace referencia directa a la Ingeniería en Comunicación Social. La propaganda es una forma de la Ingeniería en Comunicación Social, una metodología de acción sobre el sistema de comunicación social, para afectar mediante ciertas operaciones a los sistemas de información social, desde cierto tipo de sistema de información propaganda. En el caso que Eulalio Ferrer propone y defiende, ese sistema de información busca la articulación comunitaria por parte de un agente legítimo y legal, el gobierno democrático, que busca ante todo la participación de todos en una unidad de sentido en beneficio de todos.

4. Los medios de difusión colectiva tienen un papel central en la formación de la opinión pública, son instrumentos básicos de influencia y equilibrio social. Según Eulalio Ferrer en un gobierno democrático moderno serán los medios de difusión colectiva los instrumentos de operación ejecutiva de la difusión del sistema de información que tiene como efecto la construcción de un sistema de comunicación interacción colaboración.

5. Los medios de difusión colectiva representan un enorme poder, vivimos en este momento el tiempo social de su ejercicio y presencia. Los medios de difusión colectiva son percibidos por Eulalio Ferrer como el centro de la vida contemporánea en la configuración del sistema de comunicación interacción colaboración, la vida ciudadana, mediante la difusión de cierto sistema de información sintetizado para la construcción, fortalecimiento, refuerzo, de ese sistema de comunicación ciudadano.

6. La opinión pública se conforma en una fórmula que combina medios de difusión colectiva y estabilidad social. Esta fórmula es central en la apuesta del profesor Ferrer, y clave para entender su visión de la opinión pública desde la Ingeniería en Comunicación Social. Por una parte el sistema de comunicación social interacción colaboración ciudadano, y por otra parte la operación reforzadora y promotora del gobierno democrático de un sistema de información general que articula y enriquece a ese sistema de comunicación ciudadano. Esto configura un horizonte de posibilidad, un horizonte meta, una horizonte deseable, para entender y tejer una opinión pública poderosa, rica, y activa.

7. En principio los medios de difusión refuerzan la opinión pública general, y por efecto complementario modifican la pequeña, la menos profunda, la más superficial, la menos arraigada. 
Esta situación permite la percepción esquemática de una dinámica constructiva compleja. El sistema de información central de articulación de vida ciudadana, corazón de la opinión pública y de la operación básica de los medios de difusión colectiva, es reforzado una y otra vez por la acción de los medios de difusión colectiva. En forma complementaria los sistemas de información menores son articulados al sistema de información mayor. En este sentido los medios refuerzan lo general y modifican lo particular. La ingeniería en Comunicación Social es así una ejercicio constante de articulación y reforzamiento.

8. La opinión pública es articulación de fondo, la opinión del público es articulación de superficie. Este tema tiene la posibilidad constructiva de diversos escenarios con múltiples matices. Una hipótesis central sería que el vector estable y sustentable de la cultura, la matriz general articuladora de la vida social, el sistema de información ciudadano, siempre es la prioridad en el ejercicio público de la comunicación social. Todas las otras manifestaciones de comunicación social son secundarias ante esa prioridad, la que se enfoca en la articulación general del sistema de comunicación interacción colaboración ciudadano. Aún con importancias relativas a la coyuntura, las manifestaciones particulares de sistemas de información secundarios sólo serán prioritarias cuando articulan y fortalecen el sistema de información ciudadano básico general. Toda expresión es posible, todos sistema de información social es posible, pero siempre será prioritario lo que refuerza el sistema de comunicación ciudadano y el sistema de información que lo promueve y refuerza. Estas diversas relaciones contradictorias son la base de la vida democrática desde este modelo de comunicación social basado en la opinión pública.

9. El tema más agudo en los asuntos de la formación y desarrollo de la opinión pública es el de la libertad de expresión frente a la indiferencia y la apatía. La pregunta más urgente es entonces qué hacer para educar y promover la cultura de conversación necesaria para mantener vivo y con buena salud al sistema de comunicación interacción colaboración ciudadano. La Ingeniería en Comunicación Social tiene en principio en la propuesta de Eulalio Ferrer un trabajo de administración, mantenimiento, de sustentabilidad, del modelo de democracia basado en la comunicación social que supone la propuesta de opinión pública. El punto es que para llegar a ese modelo se necesita una operación tecnológica de comunicación social, y cuando se llega ahí, se necesita otro tipo de operación tecnológica de comunicación social para darle sustentabilidad en el tiempo. La primera parte del problema tiene un nombre, educación para la conversación, educación para la vida ciudadana, educación 

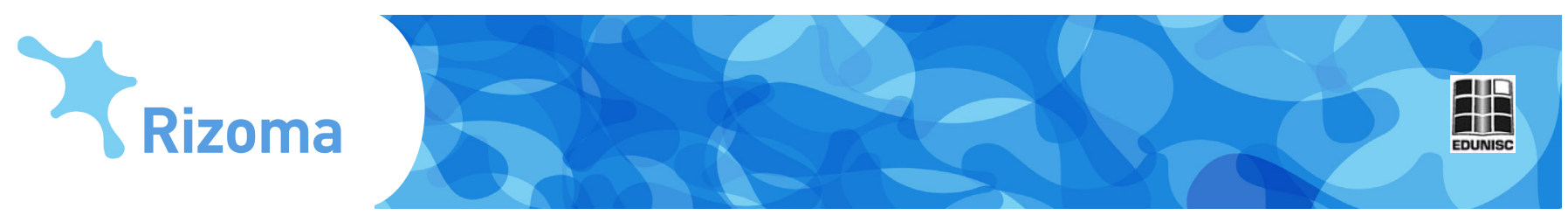

para la convivencia productiva por la comunicación interacción colaborativa. Después se trataría de mantener esa situación viva y saludable, la conversación ciudadana democrática cotidiana.

10. La opinión pública necesita de la opinión publicada, de la expresión y el debate ciudadano. La comunicación, formación de comunidad, parte de la condición elemental de la conversación. La conversación es el componente básico para el desarrollo y evolución de la opinión pública. En la conclusión de su propuesta Eulalio Ferrer sintetiza el tema tecnológico de la comunicación social, necesario para promover y preservar el ambiente democrático del sistema de comunicación social interacción colaboración ciudadano, la conversación democrática, la constante y rica conversación libre, ilustrada, comprometida y responsable. Ese sería el reto básico de la Ingeniería en Comunicación Social según el modelo de opinión pública propuesto, gestionar, promover, administrar, dar continuidad y sustentabilidad a largo plazo, a la conversación democrática ciudadana cotidiana.

El modelo de comunicación social de la opinión pública propuesta por Eulalio Ferrer tiene a la democracia en el centro, que puede ser leída por la Comunicología como un sistema de comunicación social interacción colaboración ciudadana. La Ingeniería en Comunicación Social para construir y mantener ese sistema de comunicación social supone la intervención legítima y legal del gobierno en la gestión de un sistema de información que articule a la diversidad y la pluralidad dialógica del sistema de comunicación ciudadano. La tecnología de comunicación social básica para llevar a cabo todo esto es la conversación. Y son los medios de difusión colectiva los principales instrumentos para la promoción, desarrollo y mantenimiento de dicha tecnología de comunicación social.

\section{Apunte Bibliográfico}

BAUMAN, Zygmunt (2008) Comunidad, Siglo XXI editores, Madrid.

DE KERCKHOVE, Derrick (1999) Inteligencias en conexión, Gedisa, Barcelona.

DEBRAY Régis (2001) Introducción a la mediología, Paidós, Barcelona.

DEUTSCH, Karl (1971) Los nervios del gobierno, Paidós, Buenos Aires.

ECO, Umberto (1978) Tratado de Semiótica general, Nueva imagen-Lumen, México.

ELIAS, Norbert (1987) El proceso de la civilización. Investigaciones 


\section{$\gamma_{\text {Rizoma }}$}

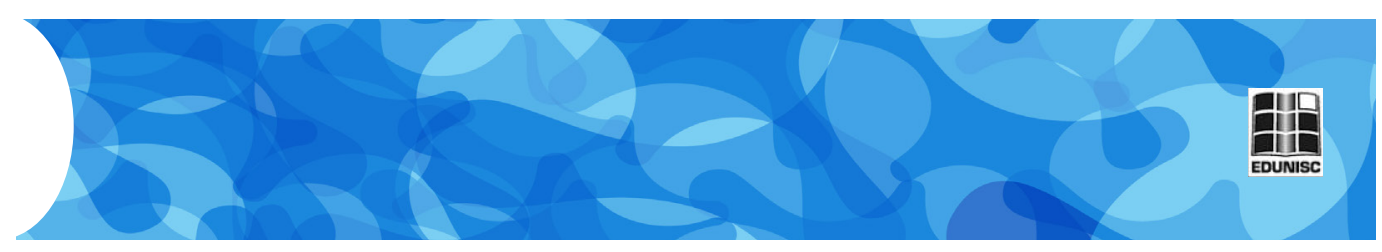

Sociogenéticas y Psicogenéticas, Fondo de Cultura Económica, Madrid.

GALINDO Cáceres, Luis Jesús (2005) Hacia una Comunicología posible, Universidad Autónoma de San Luis Potosí, San Luis Potosí.

GALINDO Cáceres, Jesús (2006) Cibercultura. Un mundo emergente y una nueva mirada, CNCA- Instituto mexiquense de la cultura, Toluca.

GALINDO Cáceres, Luis Jesús (2011) Ingeniería en Comunicación Social y Promoción Cultural. Sobre Cultura, Cibercultura y Redes Sociales, Homo Sapiens, Universidad Nacional del Rosario, Benemérita Universidad Autónoma de Puebla, Rosario.

GALINDO Cáceres, Luis Jesús (2012) Ingeniería en Comunicación Social y Deporte, Instituto de Altos Estudios en Deporte, Cultura y Sociedad, México.

GALINDO Cáceres, Luis Jesús, Tanius Karam Cárdenas y Marta Rizo

García (2009) Comunicología en Construcción. Universidad Autónoma de la Ciudad de México, México.

GALINDO Cáceres, Jesús (coordinador) (1998) Técnicas de investigación en sociedad, cultura y comunicación, Addison Wesley-Longman, México.

GALINDO Cáceres, Jesús (coordinador) (2008) Comunicación, Ciencia e Historia. Fuentes científicas históricas hacia una Comunicología Posible, McGraw Hill, Madrid.

GALINDOCáceres, Jesús (coordinador) (2009) Sociología y Comunicología. Historias y posibilidades. EDUCASA, Salta, Argentina.

GALINDO Cáceres, Jesús (coordinador) (2011b) Comunicología Posible. Hacia una ciencia de la comunicación, Universidad Intercontinental, México.

GARCÍA, Rolando (2006) Sistemas Complejos. Conceptos, métodos y fundamentación epistemológica de la investigación interdisciplinaria, Gedisa, Barcelona.

KATZ, Daniel y Robert L. Kahn (1977) Psicología social de las organizaciones, Trillas, México.

KLAPPER, J. T. (1974) Efectos de las comunicaciones de masas, Aguilar, Madrid.

KRAUSS, Sidney y Dennos Davis (1990) Comunicación Masiva, Trillas, México. 


\section{Y}

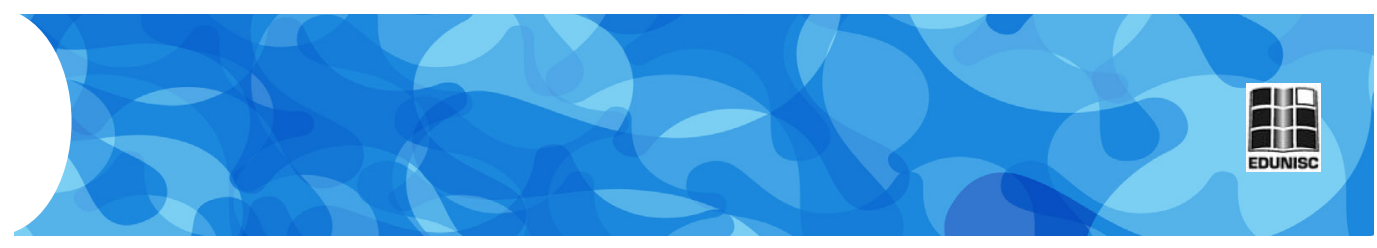

MACÍAS, Norma y Diana Cardona (2007) Comunicometodología, UIC, México.

MARTÍN Serrano, Manuel (2007) Teoría de la comunicación. La comunicación, la vida y la sociedad. McGraw Hill, Madrid.

MEAD, George Herbert (1968) Espíritu, persona y sociedad, Paidós, Buenos Aires.

RESÉNDIZ Núñez, Daniel (2008) El rompecabezas de la ingeniería. Por qué y cómo se transforma el mundo. Fondo de Cultura Económica, México.

RIFKIN, Jeremy (2010) La Civilización Empática. La carrera hacia una conciencia global en un mundo en crisis, Paidós, México.

SIMMEL Georg (2002) Sobre la individualidad y las formas sociales, Universidad Nacional de Quilmes, Buenos Aires.

TONNÏES, Ferdinand (1979) Comunidad y asociación, Península, Barcelona.

URIZPeman, María Jesús (1993) Personalidad, socialización y comunicación, Libertarias-Prodhufi, Madrid.

VON FOERSTER, Heinz (1998) Sistémica elemental, EAFIT, Medellín.

WAGENSBERG, Jorge (1994) Ideas sobre la complejidad del mundo, Tusquets, Barcelona.

WATZLAWICK, Paul et al. (1971) Teoría de la comunicación humana, Tiempo contemporáneo, Buenos Aires. 